\title{
A PERMUTATION SOLUTION TO TEST FOR TREATMENT EFFECTS IN ALTER- NATION DESIGN SINGLE-CASE EXPERIMENTS
}

\author{
Francesca Solmi ${ }^{1}$, Patrick Onghena ${ }^{2}$, Luigi Salmaso ${ }^{3}$, Isis Bulté ${ }^{2}$ \\ ${ }^{1}$ Department of Statistics, University of Padova, Italy. \\ ${ }^{2}$ Faculty of Psychology and Educational Sciences, Katholieke Universiteit of Leuven, Belgium. \\ ${ }^{3}$ Department of Management and Engineering, University of Padova, Italy.
}

Key Words: Single-case experiment, time-process, permutation test, power comparison.

\section{ABSTRACT}

Research involving a clinical intervention is normally aimed at testing the treatment effects on a dependent variable, which is assumed to be a relevant indicator of health or quality of life status. In much clinical research large- $n$ trials are in fact impractical because the availability of individuals within well-defined categories is limited in this application field. This makes it more and more important to concentrate on single-case experiments. The goal with these is to investigate the presence of a difference in the effect of the treatments considered in the study. In this setting valid inference generally cannot be made using the parametric statistical procedures that are typically used for the analysis of clinical trials and other large- $n$ designs. Hence nonparametric tools can be a valid alternative to analyze this kind of data. We propose a permutation solution to assess treatment effects in single-case experiments within alternation designs. An extension to the case of more than two treatments is also presented. A simulation study shows that the approach is both reliable under the null hypothesis and powerful under the alternative, and that it improves the performance of a considered competitor. In the end we present the results of a real case application.

\section{INTRODUCTION}

This paper is devoted to testing some treatment effects on a response variable, which can be carried out by performing a clinical experiment in which effect of several treatments on the outcome is registered. Such research can be broadly divided into relatively large-scale clinical trials and singlecase studies. In this work we concentrate on single-case experiments since often large- $n$ trials 
cannot be carried out, due to financial and human resourcing constraints. For this reason singlecase experiments are becoming more and more important.

As described in Onghena (2005), single-case experiments are characterized by the fact that one subject is observed repeatedly during a certain period of time, under different levels ("treatments") of at least one independent variable. Hence we observe only one subject (single-case, $n=1$ ), manipulating independent variable(s) (experiment); this way the subject is exposed to all levels of the independent variable, and we handle repeated measures or observations of the response variable of interest (longitudinal data, see Diggle et al. (2002)). As argued in Onghena (2005), single-case experiments have a long history in behavioral science; often they are the only viable options if rare or unique conditions are involved, and they can be motivated thinking that actually they mimic the care for the individual patient that is needed in clinical work.

The goal of single-case experiments is usually to investigate the presence of a difference in the effects of the treatments considered in the study. Instead of using standard parametric techniques, which in this setting cannot always provide valid inference, nonparametric tools can be a valid alternative to analyze this kind of data (see Todman and Dugard (2001) and Onghena (2005)). In particular, randomization of assignment of the measurement occasions to treatments allows us to find an interesting solution to the problem in permutation tests. We can thus ensure the study's internal and statistical-conclusion validity. The basic idea is to perform a randomization of the repeated observations in time, instead of in individuals as is usually done, and then choose a statistic to evaluate the difference among treatments.

In this work we aim to motivate the use of permutation techniques in order to analyze single-case data; for this reason new proposals are presented to successfully solve the problem in a permutation framework. They are shown to improve the performances of other already existing solutions. In the second Section we give a main argument for the use of randomization methods, describing how randomization is dealt with in these kinds of experiments, and hence how these methods are exact by construction, mimicking the random allocation in the real experiment. In Section 3 we describe the general approach to the solution of the testing for treatment effects in singlecase designs, underlining the need to adopt time-series analysis tools in order to model data which actually display some autocorrelation. It is worth noting that although there could be the possibility of adopting different models to fit the data such as parametric and semi-parametric models, we checked that generally such models do not provide good solutions in recognizing a general difference among the treatments, especially when the sample size is low. Section 4 is devoted to the more in depth description of a new permutation time-dependent solution, describing the general idea behind the permutation methodology for single-case experiments, and discussing proposals for the choice of best time-series analysis tools. Section 5 shows the results of a simulation study in which the proposed permutation solutions are tested under a wide variety of data scenarios, and these 
methods are applied to real clinical psychologist data in Section 6. Conclusions about the obtained results are discussed in Section 7.

\section{RANDOMIZATION IN SINGLE-CASE DESIGNS}

The starting point for correctly analyzing single-case designs is to underline the difference between random sampling and random allocation. A crucial point is understanding that random allocation does not apply exclusively to the allocation of participants to treatment conditions. In single-case designs, it also applies to the allocation of opportunities for exposure to treatment conditions. Both these points are essential to understanding that the permutation approach really does make sense in this kind of problem.

Random sampling from a large, well-defined population is the usual formal requirement for applying parametric techniques, and it justifies the generalization of results, allowing their external validity. However, it is often impractical to have a genuine random sample from a given population of interest, both because it is difficult to define the population of interest itself, and because costs and time requirements can be prohibitive. Instead, randomization in human experimentation is much more likely than random sampling; each exposure opportunity is considered to be randomly placed into separate urns, each of which represents a particular combination of experimental conditions (independent variables). Hence no general wider population is supposed to exist here, and we are able to control for confounding variables.

The simple and natural randomization mechanism considered in the study can then be used to construct a test - the permutation test - simply by mimicking this underlying random assignment. Hence the permutation test is by construction the most natural candidate for use in data analysis

following a given randomization design. Indeed we can easily construct the distribution of the test statistic under the null hypothesis of interest by randomly reassigning the condition urns to exposure opportunities.

\subsection{Different randomization designs}

The randomization designs in single-case experiments can broadly be divided into two categories alternation and phase designs (see Todman and Dugard (2001) and Onghena (2005)). The former class is characterized by the fact that any level of the independent variable can be present at each measurement occasion; hence we randomly determine the treatment sequence by taking into account the number of levels of the independent variables and the number of measurement occasions for each level. For example, let us consider the so-called completely randomize design (CRD). For one independent variable (treatment) with two levels (say $A$ and $B$ ) and three measurement 
occasions for each level, we simply have to randomly select a sequence from a number of possibilities: $A A A B B B, A A B A B B, A A B B A B, A A B B B A, A B A A B B, A B A B A B, A B A B B A, B A A B B A$, $B A A B A B, B A A A B B$, etc. Of course, depending on the specific characteristics of the study, we can impose a minimum, or a maximum, number of consecutive measurement occasions for each treatment. For instance, we can impose that a fixed number of observations under the same treatment must be recorded, and randomize the opportunities for exposure to the treatment conditions in blocks, instead of singularly. As it's name suggests, such design leaves complete freedom to the randomization of the opportunities for exposure to the treatment conditions.

Phase designs are useful when rapid and frequent alternation of treatments is difficult to perform. In this case several consecutive measurements are performed for each phase, the order of the

phases is fixed and we randomize the moments of phase change. Hence we randomly determine the treatments' phases sequence by taking into account the order of the phases, the number of treatments, the minimum length of the phases and the total length of the experiment. For example, let us consider an $A B$ design. For one independent variable (treatment) with two levels (say $A$ and $B$ ), six measurement occasions and at least one measurement for each phase, we simply have to randomly select a sequence from the following ones: $A B B B B B, A A B B B B, A A A B B B$, $A A A A B B, A A A A A B$. Notice how this randomization scheme is completely different from the CRD presented above.

\section{TESTING FOR TREATMENT EFFECTS IN SINGLE- CASE DESIGNS}

To make inference in single-case designs is not as straightforward as in large- $n$ problems. Indeed, classic parametric techniques are often not suitable for making statistical decisions while correctly controlling the inferential errors. In this work we propose a solution to test for treatment effects in single-case designs; let us use $\left\{X_{A^{j}}(t)\right\}_{t \in T}$, where $T$ is a continuous support, to indicate the time-process of the response under treatment $A^{j}$, with $j=1, \ldots, C$. C denotes the number of treatments under consideration. The null and alternative hypotheses that we are going to consider in this work can then be formalized as follows

$$
\begin{cases}H_{0}: & X_{A^{j}}(t) \stackrel{\mathcal{M}}{=} X_{A^{l}}(t) \quad \forall j<l, \quad j, l=1, \ldots, C, \quad \forall t \in T \\ H_{1}: \quad \exists j, l \in\{1, \ldots, C\}, \quad j \neq l & \mid X_{A^{j}}(t) \stackrel{\mathcal{M}}{\neq} X_{A^{l}}(t),\end{cases}
$$

where the notations $(t)$ and $\mathcal{M}$ emphasize that we are considering the model underlying the response time process. More precisely, by using $\mathcal{M}$, we aim at indicating that the equality 
assumed under the null hypothesis refers to the underlying models for the response time processes under the different treatments. Hence we aim to test the null hypothesis that the several treatments have the same effect on the time process underlying the response against the alternative hypothesis that there is at least one treatment effect which is different from the others.

Various approaches have been proposed (see Gorman and Allison (1996) for an in-depth description). Among them, time series analysis provides a valid set of procedures, such as autoregressive integrated moving average (ARIMA) models. This solution is often useful and works well, provided that we handle a sufficient number of observations.

An alternative solution is given by classic ANOVA and least squares regression approaches. Indeed, even if in principle parametric statistics require normality and homoscedasticity assumptions, in practice they are robust to violations of these assumptions. However, their robustness

is less strong in the case of small and unequal sample sizes, as actually often arises in single-case experiments. Additionally, the use of such parametric techniques can be criticized for the presence of another strong assumption - that the errors are uncorrelated. This latter problem has to be taken into account, since, as argued in Gorman and Allison (1996) and Levin et al. (1978), autocorrelation very often arises in single-case designs, and classic ANOVA and least squares regression approaches are not robust to violations of this assumption.

A third class of solutions is given by nonparametric tests. In general they represent a valid alternative to parametric solutions, especially in small- $n$ and large- $n$ cases when the required assumptions may be violated. They can be successfully applied in single-case designs; the positive results of the use of nonparametric rank tests is shown in Edgington (1996).

To summarize, as argued in Todman and Dugard (2001), for single-case designs with a large number of observations (say at least 50 per phase) the time-series analysis approach can be successfully applied. When the number of observations per phase is not that large, one may use a permutation test to solve the problem.

\section{THE PERMUTATION SOLUTION}

The permutation-based solution for single-case experiments has been discussed by many authors. A good review is given in Todman and Dugard (2001), where the authors also provide an indepth discussion of the motivations for using randomization tests in this framework. Recently, this methodology has been discussed in Bulté and Onghena (2008) and Bulté and Onghena (2009), where the description of a specific $R$ package is also given. It is also applied to real data problems in, among other works, de Jong et al. (2005) and ter Kuile et al. (2009). The basic idea is to randomize the repeated observations in time, instead of in individuals as is usually done, and then choose a statistic to evaluate the difference between the treatments. More specifically, the kind of 
randomization needed to perform the test should be based on the design aspect that is random in the experiment; hence we can randomize either the treatments at each measurement occasion, in alternation designs, or the moments of phase change, in phase designs (see Onghena (2005)).

In this paper, we propose a permutation solution to test for treatment effects in alternation single-case experiments. The aim of our research is to improve the existing proposals on the choice of test statistic to be used to compare the performance of the different treatments. For example, in Bulté and Onghena (2008) and Bulté and Onghena (2009) the authors simply use the sample mean difference, ignoring the time ordering of the observations.

In this section of the paper we firstly point out the importance of taking care of the autocorrelation between observations, and we underline how permutation tests are able to handle this problem. Then the randomization scheme and the permutation procedure itself are well defined. Furthermore, we discuss the problem of choice of test statistic, proposing the use of time-series analysis tools.

\subsection{Autocorrelation and randomization scheme}

As pointed out previously, autocorrelation, i.e. correlation between the residuals of scores in timeseries data, is of course present in single-case data. Parametric techniques such as $t$-test and ANOVA require the assumption of independence of observations, and it is known that violation of this assumption leads to higher type I errors. Therefore it is very important to take care of the serial dependence between the observations.

One main feature of randomization tests for single-case designs is that they are immune to the effect of serial dependency because under the null hypothesis there would be identical responses across occasions. As such the randomization scheme allows us to mimic the true distribution of the test statistic under the null hypothesis. However, this does not mean that any test statistic is good for making inference about the phenomenon of interest, and, if serial dependence exists among the observed data, we should choose a suitable test statistic that can capture the main feature of interest. This means that, excluding the simple case of no trend and effect level of interest, a time-series analysis tool is needed. This aspect will be treated in more depth in the next paragraph.

\subsection{Time-series solution for the test statistic}

Here we discuss the problem of choosing a suitable test statistic for testing the treatment effect in single-case designs. Readers are reminded that in Bulté and Onghena (2008) the authors propose the sample mean difference as a statistic to compare the effect of the two treatments; this choice is motivated by the argument that the focus is on an expected difference in level, which can be reflected by a difference between means. The same proposals can also be found in Bulté and 
Onghena (2009), where it is suggested that if needed, other test statistics, such as differences in slopes or intercepts, can be adopted. As far as we are aware, the problem of choosing the type of statistic has not yet been considered, and the $R$ package presented in Bulté and Onghena (2008) and Bulté and Onghena (2009) does not offer much choice. We think this is a particularly relevant topic, since the sample mean can be insufficient to distinguish between time-series: in this kind of problems the shape of the observed points in relation to time is of primary interest, as different trends can be found in different time-series while they can register the same average behavior.

The basic idea behind the development of our proposals is that a longitudinal data analysis tool is needed in this case. In order to provide a completely nonparametric solution, nonparametric tools may seem more appropriate. It is true, however, that even using a parametric tool on each randomized sample, then using its permutation distribution, might be a solution given that we do in fact treat the resulting statistic as a simple choice for the test statistic, completely disregarding its inferential properties.

More precisely, our idea is to estimate the time functional shape of observations (with a nonparametric smoother (see Simonoff (1996)), as splines, kernel smoother, local regression, as well as a parametric time-series analysis tool) and then use a summary statistic of the resulting curve (say $\psi(\cdot))$. By randomizing the observations according to the randomization design, we are able to reproduce the distribution of the test statistic under the null hypothesis, and hence evaluate the p-value for the test of interest. Our proposal is to estimate the time functional shape of observations for the sub-datasets of various treatments, and then use a summary statistic which is able to measure the difference between these smoothed time-processes. Indeed, under the null hypothesis the outcome variable should follow similarly shaped time processes for the different treatments; hence by randomizing the labels of the treatment in order to construct the permutation distribution of the test statistic, we expect to end up with a set of low values for the difference between the various smoothed time-series. We consider large values of the test statistics as evidence against the null hypothesis.

Formally, we can describe the general randomization procedure as follows:

i. consider the original time-series $x=\left(x_{1}, \ldots, x_{T}\right)$, and compute the observed value for the test statistic $t^{o b s}=\psi(x)$;

ii. according to the randomization scheme, perform a randomization of the treatments' labels for $x$, obtaining the permuted time-series $x^{*, 1}$, and compute the value for the test statistic $t^{*, 1}=\psi\left(x^{*, 1}\right)$

iii. independently repeat Step ii. B times, getting the values of the test statistic $t^{*, i}=\psi\left(x^{*, i}\right)$, for $i=1, \ldots, B$; 
iv. construct the permutation distribution of the test statistic under the null hypothesis from the vector of values $t^{*}=\left(t^{*, 1}, \ldots, t^{*, B}\right)$;

v. compute the p-value of the test for treatment effects, for example rejecting the null hypothesis for high values of the test statistic, as $p=\sum_{i=1}^{B} \mathbf{1}_{\left(t^{o b s}, \infty\right)}\left(t^{*, i}\left(x^{*, i}\right)\right) / B$, where $\mathbf{1}_{(\cdot, \cdot)}(\cdot)$ denotes the indicator function.

We remark that $B$ can be either the number of all the possible permutations of the data (the whole permutation space) or a lower number if we are interested in using only a Monte Carlo sample of them (for further details see Pesarin and Salmaso (2010)). In the following section we provide a more in-depth description of the proposed randomization solution.

\subsection{Formalization of the procedure}

In alternation designs each treatment can arise at any time occasion in the single-case experiment. Once the treatment sequence is randomized, we handle a time process in which we alternate the considered treatments. As already pointed out, we propose to separately smooth the different treatment-labelled time-processes, and then use a measure of the distance between the curves as a test statistic. Firstly we define the concept of exchangeability under the null hypothesis when the underlying model for the response time-process is of interest. Then we discuss both the choice of smoother and of test statistic.

Let us use $f$ to denote the density of response $X, f^{(n)}(x)$ to denote the density of the sampling variable $X^{(n)}$, and $x=\left(x_{1}, \ldots, x_{n}\right)$ to denote the dataset. In general the exchangeability of the observed data with respect to groups under the null hypothesis is said to hold if $f^{(n)}\left(x_{1}, \ldots, x_{n}\right)$ $=f^{(n)}\left(x_{u_{1}^{*}}, \ldots, x_{u_{n}^{*}}\right)$, where $\left(u_{1}^{*}, \ldots, u_{n}^{*}\right)$ is any permutation of $(1, \ldots, n)$. We can adapt this characterization to the case of time-dependent data as follows: Firstly we introduce time into the formulation, and substitute the concept of 'density' with that of 'model'. Thus we speak about the model underlying the sampling variable time-process $\mathcal{M}^{(n)}(x ; t)$. We then recall that in the single-case framework, and in particular in the case of alternation designs, the experimental random quantity is the treatment assignment. Hence we can say that the exchangeability of the observed data with respect to treatments under the null hypothesis is said to hold if $\mathcal{M}^{(n)}\left(x_{1}, \ldots, x_{n} ; t\right)=$ $\mathcal{M}^{(n)}\left(x_{u_{1}^{*}}, \ldots, x_{u_{n}^{*}} ; t\right)$, where $\left(u_{1}^{*}, \ldots, u_{n}^{*}\right)$ is any permutation of the original treatment sequence.

As regards the choice of a smoother, the best candidate, or best time-series modeling tool in general, of course depends on the real data being handled. The same holds for the choice of most suitable test statistic. An interesting feature of permutation tests is that in principle we can choose any statistic depending on the data we wish to analyze and the study's objective. Moreover, the opinion of the expert who presents the problem can also be taken into consideration in making this choice. If, for example, the expert expects that all treatments will lead to a trend in the 
outcome, and that what is of interest is the difference between the slopes and the intercepts of these trends, then we should choose a time-series analysis tool capable of taking into account the underlying slopes and intercepts of the different treatment trends. Therefore the nature of the data is also important. The number of observations per treatment for example can also help us to decide a suitable smoother. We strongly believe that several implicit and explicit aspects of the data itself should be taken into account when choosing the test statistic. In this work we propose some possible choices, which of course do not exclude the possibility of applying different ones depending on specific real data features. However, we aim to provide a unique main proposal both for the smoother and the test statistic, which should be better able to detect treatment effects for any feature of the data than the others, at least in the field of educational and behavioral sciences. Two categories of techniques for modeling time-series data are nonparametric smoothers and classical ARIMA models. Nonparametric smoothers can be a good choice when we have no idea what the time-process underlying the data is (for instance when we handle an experiment concerning a new treatment, and not even the field expert knows what to expect from the data). Instead, ARIMA modeling can be a useful tool when pilot studies are available, or at least previous analyses on similar problems. In the following we will consider only the former class of models, for one main reason: ARIMA models are only applicable with equispaced time-series data, which poses a problem for the solution we are proposing. Indeed, since each treatment can arise in every time occasion, then each single treatment time-series does not in general have equispaced observations across time. Nonparametric smoothing is instead particularly suitable for our purposes. We need to somehow measure the distance between the several treatment curves, hence nice objects we can handle are continuous curves to compare, which actually is the output of smoothers. Among nonparametric smoothers many choices exist, such as splines, kernel smoothers and local regression. As a starting point, we consider local regression to be a good choice. It is a widely accepted method, it gives good fitting results even with relatively short time-series, and it is quite simple to understand even for users out of the statistical world.

As regards the choice of test statistics, it should, as already said, be a measure of the distance between the curves. Several choices can be made and, according to Fisher (1935), in principle there is no preferable statistic for all data features. Depending on different features of the same null hypothesis, a list of different test statistics may be more appropriate. This leads to the MultiAspect (MA) testing issue (see Pesarin (2001)). Therefore in our particular framework, we propose to nonparametrically combine several test statistics $t_{m}^{\prime}(x)$, for $m=1, \ldots, M$, about which more details will be given in Section 5 .

The permutation procedure for alternation designs can be summarized as follows: Let us consider the general case of $C$ treatments, denoted by $A^{1}, A^{2}, \ldots, A^{C}$; also let $s_{A^{j}}\left(t ; x_{A^{j}}\right)$, for $j=1, \ldots, C$, be the resulting local regression smoothed processes from, respectively, data labelled 
with treatments $A^{j}$, for $j=1, \ldots, C ; s_{A^{j}}\left(t ; x_{A^{j}}\right)$ emphasizes the fact that the smoother is a function of time $t$, hence defined in every time occasion, given the sub-series $x_{A^{j}}$ only. Then:

i. consider the original time-series $x=\left(x_{1}, \ldots, x_{T}\right)$, and the $C$ sub-series $x_{A^{j}}=\left(x_{A^{j}, 1_{A^{j}}}, \ldots, x_{A^{j}, T_{A^{j}}}\right)$. Compute the $C$ smoothed processes $s_{A^{j}}\left(t ; x_{A^{j}}\right)$, for $j=1, \ldots, C$;

ii. compute the observed value for the partial test statistics $t_{m}^{\prime},{ }^{o b s}(x)=\psi_{m}(x)=\psi_{m}\left(s_{A^{1}}\left(t ; x_{A^{1}}\right), \ldots, s_{A^{C}}\left(t ; x_{A^{C}}\right)\right)$, for $m=1, \ldots, M ;$

iii. according to the randomization scheme, perform a randomization of the treatments' labels for $x$, getting the randomized time-series $x^{*, 1}$ and the $C$ permuted sub-series $x_{A^{j}}^{*, 1}$. Compute the $C$ permuted smoothed processes $s_{A^{j}}^{* 1}\left(t ; x_{A^{j}}^{*, 1}\right)$, for $j=1, \ldots, C$;

iv. compute the value of the partial test statistics $t_{m}^{\prime},{ }^{*, 1}=\psi_{m}\left(x^{*, 1}\right)=\psi_{m}\left(s_{A^{1}}^{*, 1}\left(t ; x_{A^{1}}^{*, 1}\right), \ldots, s_{A^{C}}^{*, 1}\left(t ; x_{A^{C}}^{*, 1}\right)\right)$, for $m=1, \ldots, M$

v. repeat Steps iii. and iv. B times, getting the values of the partial test statistics $t_{m}^{\prime *, i}=$ $\psi_{m}\left(x^{*, i}\right)=\psi_{m}\left(s_{A^{1}}^{*, i}\left(t ; x_{A^{1}}^{*, i}\right), \ldots, s_{A^{C}}^{*, i}\left(t ; x_{A^{C}}^{*, i}\right)\right)$, for $i=1, \ldots, B, m=1, \ldots, M$

vi. construct the permutation distributions of the partial test statistics under the null hypothesis from the vectors of values $t_{m}^{\prime, *}=\left(t_{m}^{\prime, *, 1}, \ldots, t_{m}^{\prime, *, B}\right)$, for $m=1, \ldots, M$;

vii. nonparametrically combine the partial permutation tests, obtaining the permutation distribution of the Multi-Aspect global test $t_{M A}^{\prime \prime}=\Psi\left(t_{1}^{\prime}, \ldots, t_{M}^{\prime}\right)$;

viii. compute the p-value of the global Multi-Aspect test for treatment effects, for example rejecting the null hypothesis for high values of the test statistic, as $p=\sum_{i=1}^{B} \mathbf{1}_{\left(t^{\prime \prime}, o b s, \infty\right)}\left(t^{\prime \prime}, *, i\left(x^{*, i}\right)\right) / B$.

\section{SIMULATION STUDY}

A Monte Carlo simulation study was performed to demonstrate the reliability and power of the proposed procedures in single-case experiments with univariate responses, in the case of a bidirectional alternative hypothesis. The study was divided into two main parts. Firstly we considered the simplest case of $C=2$ treatments, where the tested hypotheses are

$$
\left\{\begin{aligned}
H_{0}: & X_{A^{1}}(t) \stackrel{\mathcal{M}}{=} X_{A^{2}}(t) \quad \forall t \in T \\
H_{1}: & X_{A^{1}}(t) \stackrel{\mathcal{M}}{\neq} X_{A^{2}}(t),
\end{aligned}\right.
$$


Secondly we considered the more general case of $C>2$ treatments. The reliability of the methods was measured through the estimation of the type I error, while the capability of the procedures to detect treatment effects was deduced from the estimation of power. In the latter case different data scenarios were simulated under the alternative hypothesis, considering several underlying models for the treatment time-processes. We compared the new permutation solutions with the proposal in Bulté and Onghena (2008). As regards the smoother, we performed a local polynomial regression to degree 2 , span parameter $\lambda=0.75$ and tricubic weighting (proportional to $\left.\left(1-(\text { dist } / \text { maxdist })^{3}\right)^{3}\right)$.

In the case of $C=2$, we propose two $M A$ solutions which use the following partial test statistics. The first global test (hereafter labeled $t_{M A_{1}}^{\prime \prime}$ ) is constructed combining the partial tests

$$
\begin{aligned}
t_{1}^{\prime}(x) & =\frac{\sum_{j=1}^{l_{\text {grid }}}\left(s_{A^{1}}^{*, i}\left(t_{j} ; x_{A^{1}}^{*, i}\right)-s_{A^{2}}^{*, i}\left(t_{j} ; x_{A^{2}}^{*, i}\right)\right)^{2}}{l_{\text {grid }}} \\
t_{2}^{\prime}(x) & =\max _{j=1, \ldots, l_{\text {grid }}}\left(\left|s_{A^{1}}^{*, i}\left(t_{j} ; x_{A^{1}}^{*, i}\right)-s_{A^{2}}^{*, i}\left(t_{j} ; x_{A^{2}}^{*, i}\right)\right|\right) .
\end{aligned}
$$

Notice that $t_{1}^{\prime}$ is a proxy of the area between the two smoothed time sub-processes. In practice this quantity has been approximated by the mean of the squared distances on a regular time grid $t_{1}, \ldots, t_{l_{\text {grid }}}$ of length $l_{\text {grid }}<100$. Instead $t_{2}^{\prime}$ is the well-known two-sample Kolmogorov-Smirnov type statistic. The second global test (hereafter labeled $t_{M A_{2}}^{\prime \prime}$ ) is constructed combining the above presented partial tests with the one proposed in Bulté and Onghena (2008) (hereafter denoted by $\left.t_{B O}\right)$, which uses the absolute value of the difference between the sample means in the two treatment groups. In order to increase the procedure's power, we propose to use Tippett combining function (see Pesarin (2001) and Pesarin and Salmaso (2010)), given that under the alternative hypothesis it chooses the smallest partial p-value. Hence

$$
t_{M A_{k}}^{\prime \prime}(x)=\Psi\left(t_{1}^{\prime}, \ldots, t_{M_{k}}^{\prime}\right)=\max \left(1-\lambda_{1}^{\prime}, \ldots, 1-\lambda_{M_{k}}^{\prime}\right)
$$

where $\lambda_{m}^{\prime}, m=1, \ldots, M_{k}$, indicates the permutation p-value of the $m$ th partial test, so in our case $k \in\{1,2\}$ and $M_{k} \in\{2,3\}$.

The simulation study was performed generating $M C=1000$ samples from the assumed underlying models; for the implementation of the permutation tests we used $B=1000$ permutations (drawing a Monte Carlo random sample from the permutation space). We considered errors coming from both the standard normal and the Student $t$ distribution with 2 degrees of freedom. We considered four lengths for the entire time series, $n=30,50,70,100$, and five underlying ARIMA models, $A R(1), M A(1), A R M A(1,1)$, and $A R I M A(1,1,1)$ with and without constant term $\mu=1$, each with several values for the autoregressive and moving-average parameters, $\phi, \theta=-0.3,-0.2,-0.1,0,0.1,0.2,0.3,0.4,0.5$. For the normal errors case, the results showed the reliability of the proposed methods under the null hypothesis. 
The power of the procedures was also investigated under different data scenarios. Again we considered four lengths for the entire time series, $n=30,50,70,100$, and generated the difference in treatment effects by adding two different deterministic parts to the same underlying stochastic ARMA models. The rationale behind this choice is that we expect a patient's response to different treatments to remain the same in the underlying autocorrelation structure, and change only in the deterministic component of the model. In what follows we will denote the different treatments with the letters $A, B, C, D, E$. More precisely, data have been simulated as follows:

$$
\begin{aligned}
y_{t} & =\text { deterministic component }+ \text { stochastic component } \\
& =\left\{\begin{array}{ll}
f_{A}\left(y_{t-1}\right)+\phi y_{t-1}+\theta \epsilon_{t-1}+\epsilon_{t} & \text { if } \text { Treatment }_{t}=A \\
f_{B}\left(y_{t-1}\right)+\phi y_{t-1}+\theta \epsilon_{t-1}+\epsilon_{t} & \text { if } \text { Treatment }_{t}=B
\end{array},\right.
\end{aligned}
$$

with $\epsilon_{t} \sim N(0,1)$ or $\epsilon_{t} \sim t_{2}$ iid. As regards the deterministic part of the model (i.e. the fixed effects' part), we simulated treatment $A$ as both a placebo and an active treatment, and treatment $B$ as the active treatment, mimicking both a difference in level and slope of the trend between the two treatment effects. We simulated a difference in levels between the treatments as follows:

$$
y_{t}=\left\{\begin{array}{ll}
\phi y_{t-1}+\theta \epsilon_{t-1}+\epsilon_{t} & \text { if } \text { Treatment }_{t}=A \\
\delta+\phi y_{t-1}+\theta \epsilon_{t-1}+\epsilon_{t} & \text { if } \text { Treatment }_{t}=B
\end{array} .\right.
$$

Three ARMA models, $A R(1), M A(1)$ and $A R M A(1,1)$ were considered, each with several values for the autoregressive and moving-average parameters, $\phi, \theta=-0.3,-0.1,0.1,0.3,0.5$. Additionally, several values were considered for the level differences $\delta=1,3,5,10$. Therefore we simulated a difference in the slope of the trend between treatments as follows:

$$
y_{t}=\left\{\begin{array}{cc}
\beta_{A}+y_{t-1}+\epsilon_{t} & \text { if } \text { Treatment }_{t}=A \\
\beta_{B}+y_{t-1}+\epsilon_{t} & \text { if } \text { Treatment }_{t}=B
\end{array} .\right.
$$

Notice that treatment $A$ mimics either a 'no treatment' setting when $\beta_{A}=0$ or an active treatment when $\beta_{A} \neq 0$, while treatment $B$ always has a linear trend in the response. Again several values have been considered for the slope differences combining different values for the slopes $\beta_{A}=-50,-30,-10,-5,-1,0,1$ and $\beta_{B}=1,5,10,30,50$. Some of the results for the normal errors case are displayed in Figures 1 and 2 for the case of a treatment difference in level and a treatment difference in slope respectively. While $t_{B O}$ shows greater power when the two treatments are simulated with different levels, it performs worse when we consider a difference in slopes; in the latter case the estimated rejection probability under the alternative hypothesis is greater than the nominal level $\alpha$ only when the slopes of the two treatments have opposite signs. Regarding the $M A$ solutions, $t_{M A_{2}}^{\prime \prime}$ performs better than $t_{M A_{1}}^{\prime \prime}$ in the case of a difference in levels, but not a difference in slopes, where $t_{M A_{1}}^{\prime \prime}$ often reports greater power. These results are coherent with the 
way in which the MA solutions are constructed. In $t_{M A_{2}}^{\prime \prime}$, use of the partial test on the difference between the sample means allows us to increase power when the treatments differ in level, but it decreases in the case of different slopes. It should therefore be noted that in the case of different slopes, $t_{M A_{2}}^{\prime \prime}$ is always more powerful than $t_{B O}$. In the end, the power of all procedures increases with the number of observations and with the difference between the deterministic parts of the two models. Moreover, the power values reach 1 as $n$ and $\delta$ increase when we simulate differences in levels, while this is not the case for differences in slopes, where the power function seems to have a horizontal asymptote below 1.

Regarding the effect of the autocorrelation on the power behavior of the proposed methods, in general the estimated rejection probabilities change when changing the autocorrelation values. They slightly decrease as the autocorrelation parameters of the ARMA models (it does not matter which model is considered) move, in absolute value, away from zero.

Similar results have been obtained for the case of errors generated from the $t_{2}$ distribution. All the procedures control the type-I error at the nominal level; the power simply decreases with respect to the normal errors case, but the behavior of the three procedures remains the same.

Considering the more general case of $C>2$ treatments, the new solution is built performing the $M A$ permutation tests on all pairwise comparisons between treatments, and then combining them in a global test. Formally, for $i=1, \ldots, B$, the $i$ th permutation value of the test is constructed as follows:

$$
\begin{aligned}
t^{*, i} & =\psi\left(s_{A^{1}}^{*, i}\left(t ; x_{A^{1}}^{*, i}\right), \ldots, s_{A^{C}}^{*, i}\left(t ; x_{A^{C}}^{*, i}\right)\right) \\
& =\sum_{l<s, l, s \in\{1, \ldots, C\}} \frac{t_{M A_{k} ; l, s}^{\prime \prime, * i}(x)}{s d\left(t_{M A_{k} ; l, s}^{\prime \prime, *}(x)\right)},
\end{aligned}
$$

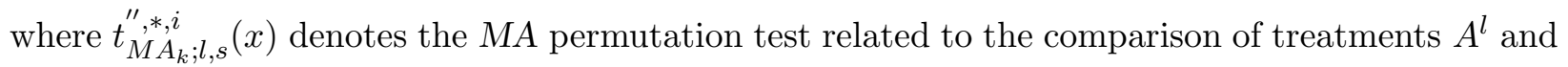
$A^{s}$. Notice that the procedure coincides with operating a nonparametric combination of the $M A$ pairwise comparisons permutation tests using a Direct combining function (see Pesarin (2001) and Pesarin and Salmaso (2010)). In the summation we divide the terms by their standard deviation in order to standardize the combined test statistics.

Again we worked with $C M C=1000, B=1000$, and the cases of $C=3$ and $C=5$ were considered. For $C=3$ and $C=5$, as lengths we simulated for the entire time series respectively $n=45,75,150$ and $n=75,125,250$ and three underlying ARMA models, $A R(1), M A(1)$ and $\operatorname{ARMA}(1,1)$, each with several values for the autoregressive and moving-average parameters, $\phi, \theta=$ $-0.3,-0.2,-0.1,0,0.1,0.2,0.3,0.4,0.5$.

The results confirm the reliability of the proposed solutions, showing the control of the type-I error at the nominal level $\alpha=0.05$. We performed a simulation study to show that the power of the MA solutions increases with sample size, distance from the null hypothesis and number of 

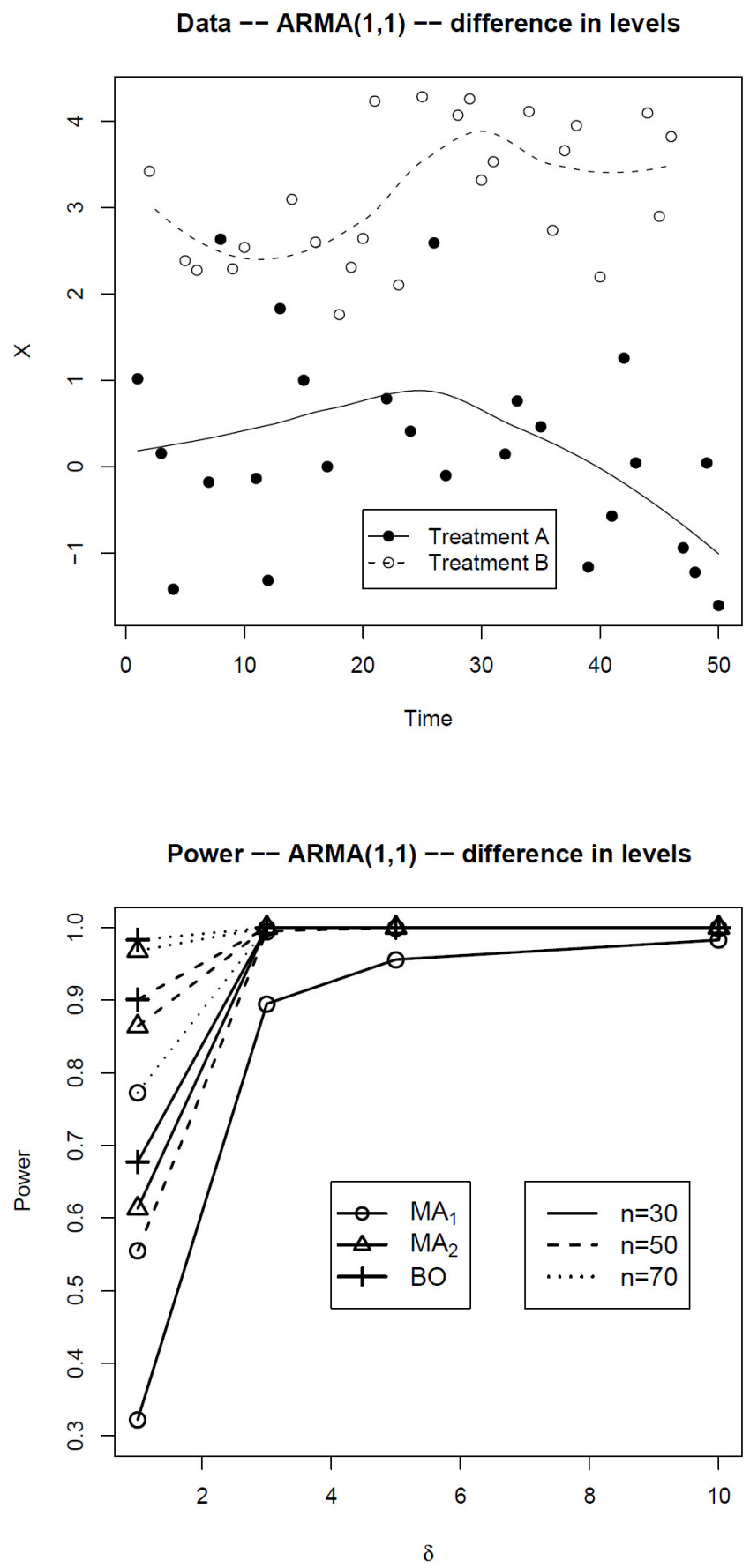

Figure 1: Simulation study. Above an example of simulated data with a difference between levels with an $A R M A(1,1)$ model for the errors with parameters $\phi, \theta=0.1, n=50$ and $\delta=3$; below power functions for the three considered permutation solutions in the case of an $A R M A(1,1)$ model for the errors with parameters $\phi, \theta=0.1$, while $n$ and $\delta$ are varying. 

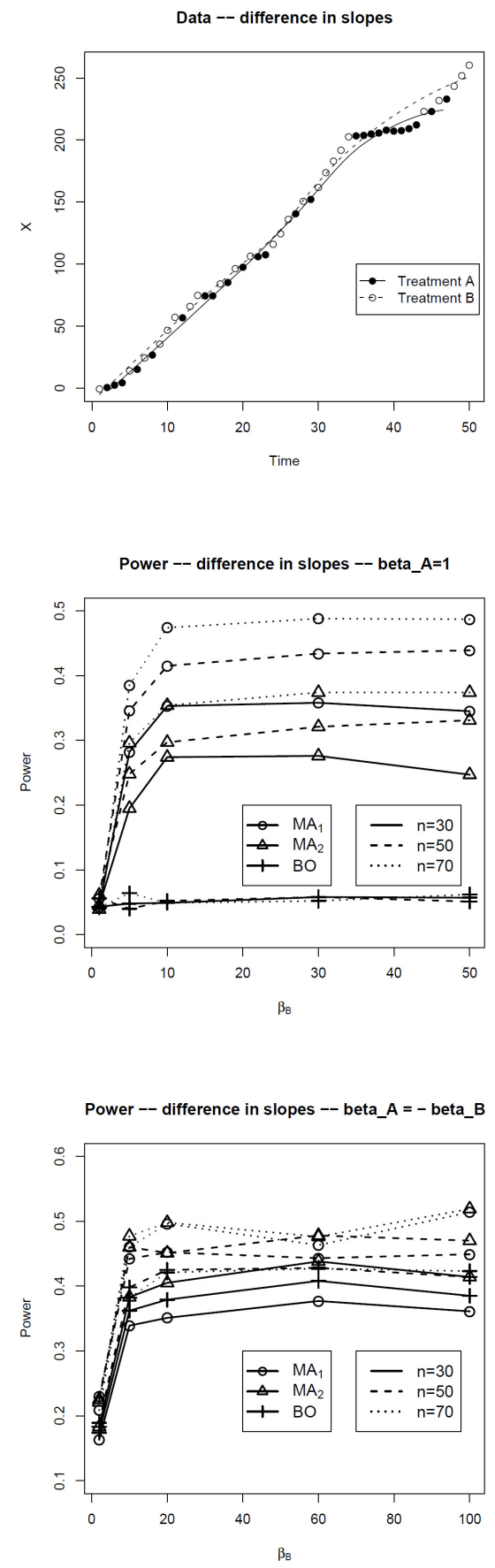

Figure 2: Simulation study. At the top an example of simulated data with a difference between slopes with $n=50, \beta_{A}=1$ and $\beta_{B}=10$; in the middle power functions for the three considered permutation solutions in the case of $\beta_{A}=1$ while $n$ and $\beta_{B}$ are varying; below power functions for the three considered permutation solutions in the case of $\beta_{A}=-\beta_{B}$ while $n$ and $\beta_{B}$ are varying. 
pairwise comparisons under the alternative hypothesis. As with the case of $C=2$, we generated the treatment effect adding different treatments to the data generating processes and different deterministic parts to the same stochastic underlying ARMA models. We considered both a difference in level and slope of the trend among the several treatment effects, following a similar scheme to the one used for $C=2$. We simulated a difference in levels among treatments considering three ARMA models, $A R(1), M A(1)$ and $A R M A(1,1)$, each with several values for the autoregressive and moving-average parameters, $\phi, \theta=-0.3,-0.1,0.1,0.3,0.5$. Moreover, for both the cases of $C=3$ and $C=5$, four settings were considered for the level differences, which are described in Table 1. The settings differ both in terms of the total difference among the treatment levels $\left(\delta_{t o t}\right)$ and the number of false null hypotheses in the family of all pairwise comparisons.

Table 1: Settings of treatments' levels $\delta_{j}, j \in\{A, B, C, D, E\}$; cases of $C=3$ and $C=5$.

\begin{tabular}{c|c|cc|ccccc}
$C$ & Setting & $\delta_{\text {tot }}$ & False $H_{0}$ & $\delta_{A}$ & $\delta_{B}$ & $\delta_{C}$ & $\delta_{D}$ & $\delta_{E}$ \\
\hline \hline \multirow{5}{*}{3} & 1 & 2 & $2 / 3$ & 0 & 0 & 1 & & \\
& 2 & 4 & $3 / 3$ & 0 & 1 & 2 & & \\
& 3 & 4 & $2 / 3$ & 0 & 0 & 2 & & \\
& 4 & 8 & $3 / 3$ & 0 & 2 & 4 & & \\
\hline \multirow{5}{*}{5} & 5 & 4 & $4 / 10$ & 0 & 0 & 0 & 0 & 1 \\
& 6 & 10 & $7 / 10$ & 0 & 0 & 0 & 1 & 2 \\
& 7 & 8 & $4 / 10$ & 0 & 0 & 0 & 0 & 2 \\
& 8 & 20 & $7 / 10$ & 0 & 0 & 0 & 2 & 4 \\
\hline
\end{tabular}

Some of the results for the case of $C=3$ are displayed in Figure 3. Notice that the power of both $M A$ solutions increases with sample size; furthermore, we obtain a higher power when we also increase the distance from the global null hypothesis (in Figure 3 summarized as a combination between the number of false partial null hypotheses and the total difference among the treatment levels, $\left.\delta_{\text {tot }}\right)$; this behavior is less clear in the case of $C=5$, probably due to the coexistence of too many different deterministic parts in the full time series, which makes it more difficult for the tests to distinguish between different effects.

We simulated a difference in slopes among treatments considering four settings for both the cases of $C=3$ and $C=5$, described in Table 2: the settings differ both in terms of the total difference among the treatment slopes $\left(\beta_{t o t}\right)$ and the number of false null hypotheses in the family of all pairwise comparisons. Again in this case the power of both $M A$ solutions increases with sample size; furthermore, we obtain a higher power when we also increase the number of false null hypotheses in the family, even if this behavior is less clear than in the case of a difference in levels among the treatment effects. Again we find a less clear increase of power in the case of $C=5$.

In the end, in the case of both a difference in levels and a difference in slopes among the treatments, it has to be underlined that the order between the power of the two MA solutions 


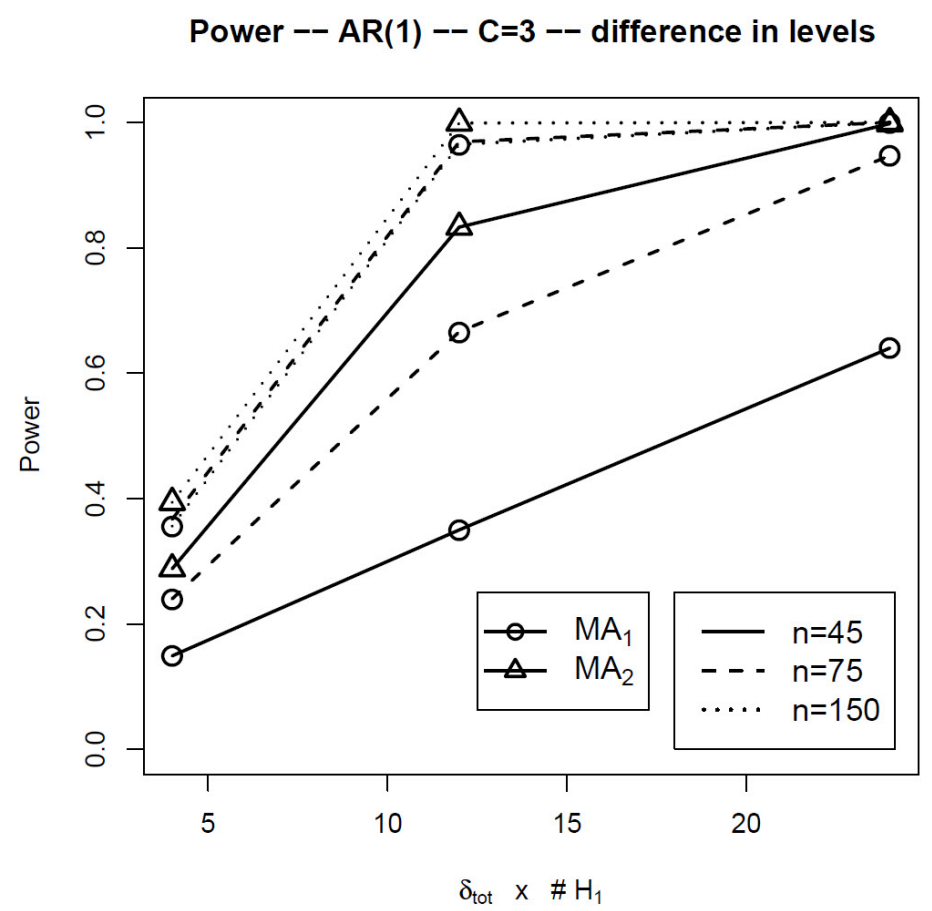

Figure 3: Simulation study. Power functions for the two considered MA solutions in the case of $C=3$, an $A R M A(1,1)$ model for the errors with parameters $\phi, \theta=0.1$, while $n$, $\delta_{\text {tot }}$ and the number of false partial null hypotheses are varying.

Table 2: Settings of treatments' slopes, cases of $C=3$ and $C=5$.

\begin{tabular}{c|c|cc|ccccc}
$C$ & Setting & $\beta_{\text {tot }}$ & False $H_{0}$ & $\beta_{A}$ & $\beta_{B}$ & $\beta_{C}$ & $\beta_{D}$ & $\beta_{E}$ \\
\hline \hline \multirow{4}{*}{3} & 1 & 4 & $2 / 3$ & 1 & 1 & 5 & & \\
& 2 & 18 & $3 / 3$ & 1 & 5 & 10 & & \\
& 3 & 18 & $2 / 3$ & 1 & 1 & 10 & & \\
& 4 & 48 & $3 / 3$ & 1 & 10 & 30 & & \\
\hline \multirow{5}{*}{5} & 5 & 16 & $4 / 10$ & 1 & 1 & 1 & 1 & 5 \\
& 6 & 44 & $7 / 10$ & 1 & 1 & 1 & 5 & 10 \\
& 7 & 36 & $4 / 10$ & 1 & 1 & 1 & 1 & 10 \\
& 8 & 134 & $7 / 10$ & 1 & 1 & 1 & 10 & 30 \\
\hline
\end{tabular}

is the same as the order obtained in the related simulation settings for the case of $C=2$. The coherence between the simple case and the generalization to $C>2$ is of course a good feature of the method. Regarding the effect of the autocorrelation on the power behavior, we can notice a similar behavior in case of $C=2$ treatments: the results show a decrease of the estimated rejection probabilities as the autocorrelation parameters move, in absolute value, away from zero. 


\section{REAL CLINICAL PSYCHOLOGIST DATA APPLI- CATIONS}

We also applied the proposed MA solutions to real data from a single-case experiment carried out at the Virga Jesse Hospital in Hasselt, Belgium (see Baplu (2005)). The data were collected from a 17-year old adolescent who had concentration problems, but who did not meet the formal DSM-IV-TR criteria for Attention Deficit Disorder (ADD). The patient came to consultation with his mother and complained about increasing attention problems at school and worsening of his school grades. They had heard about the effect of methylphenidate (Ritalin) on concentration and wondered whether they could obtain a prescription.

Although there is some evidence that Ritalin has a positive effect on concentration, even in the absence of ADD, the general use of this drug for mild conditions (or to improve intellectual performance) is still very controversial, and the effect could vary strongly from individual to individual (see Agay et al. (2010) and Crutchley and Temlett (1999)). Therefore, the physician, in close collaboration with a researcher from the K.U.Leuven, proposed to design a single-case experiment to determine the effectiveness (and also the possible side-effects) of this drug, and only to continue use of the drug after convincing experimental results.

The experiment was approved by the Ethics Committee of the hospital and the patient and his parents gave informed consent including a detailed account of the purpose, risks, and design of the study. The study was set up as a randomized, double-blind, placebo-controlled singlecase experiment, comparing Ritalin to placebo. The study was randomized because six treatment

periods and six placebo periods were randomly assigned to twelve available time blocks (i.e., one treatment order was randomly selected from $12 ! / 6 ! 6 !=924$ possibilities). A time block consisted of two days. Because Ritalin washes out within five hours, no carryover effects from one day to the next were expected (see Tannock et al. (1989)).

The study was double-blind because neither the patient and his family, nor the physician and the researcher knew the actual treatment order. A sealed envelope containing the actual treatment order was prepared by the researcher's supervisor and handed directly to the hospital pharmacist who prepared identical capsules (same shape, color, and taste) for the active medication and the placebo. The capsules had to be taken twice a day, and also the primary outcome measure was taken twice a day. Because a time block consisted of two days, this means that four measurements were available for each treatment period as well as for each placebo period.

The outcome measure was the sum of three scores on items from the ADHD Rating Scale IV, which were selected as most relevant by the patient, his parents, and the physician. The patient gave a score on an anchored scale from 0 (never or rarely) to 3 (very often) in a diary for each of the following items: 
- Does not follow through on instructions and fails to finish work.

- Fails to give close attention to details or makes careless mistakes in schoolwork.

- Has difficulty sustaining attention in tasks or play activities.

In the diary also more general comments, other complaints and potential adverse or side effects could be registered.

Figure 4 shows the summed scores of these three items. We wish to highlight the fact that the same application was considered in Bulté and Onghena (2008), where a directional alternative hypothesis was considered. We performed the two $M A$ proposals $\left(t_{M A_{1}}^{\prime \prime}\right.$ and $\left.t_{M A_{2}}^{\prime \prime}\right)$ and the $t_{B O}$

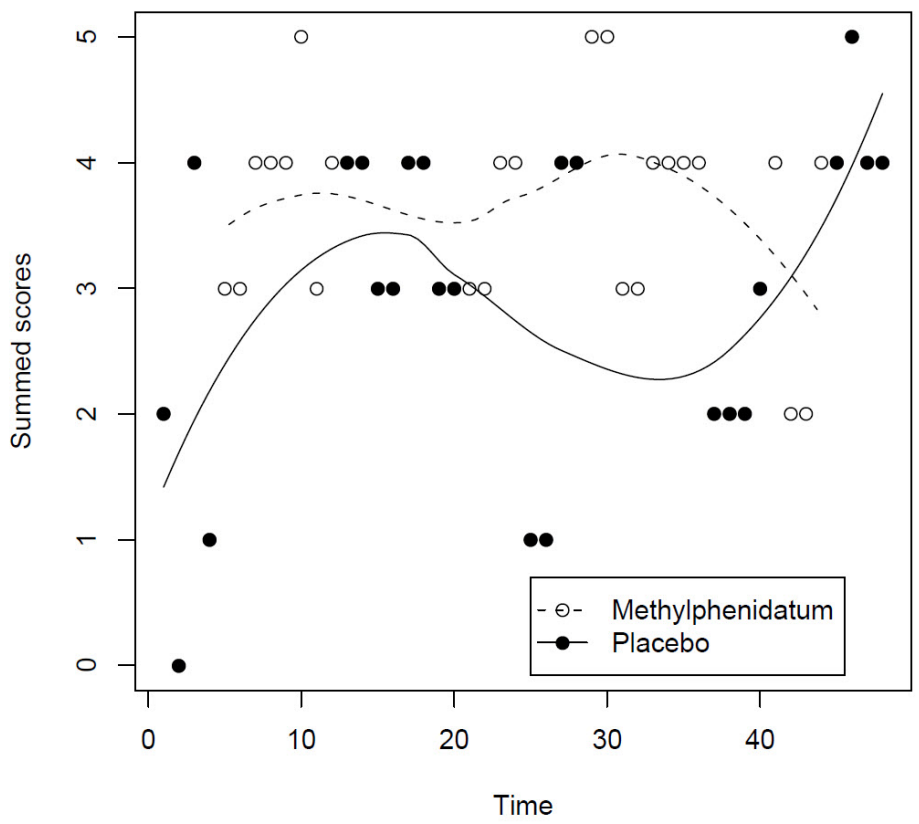

Figure 4: Real data from Baplu (2005): summed scores and smoothed time sub-processes.

solution; the results are shown in Table 3. A visual inspection of Figure 4 might suggest that the

Table 3: P-values of the tests on the difference between placebo and active drug effects; data from Baplu (2005).

\begin{tabular}{c|ccc} 
& $t_{M A_{1}}^{\prime \prime}, l_{\text {grid }}=100$ & $t_{M A_{2}}^{\prime \prime}, l_{\text {grid }}=100$ & $t_{B O}$ \\
\hline P-values & 0.0997 & 0.1430 & 0.1105 \\
\hline
\end{tabular}


intake of Rilatine results in better concentration scores as compared to placebo, but the permutation test shows that there is only weak evidence against the null hypothesis. The three test statistics

provide p-values around $10 \%$, with $t_{M A_{1}}^{\prime \prime}$ providing the smallest p-value and $t_{M A_{2}}^{\prime \prime}$ providing the largest p-value. On the basis of his results the adolescent decided not to continue the medication. Furthermore, in a debriefing interview after the study, it was clear that he could not discriminate between the treatment and the placebo periods.

\section{CONCLUSIONS}

In this work we present a permutation solution to test for treatment effects in single-case experiments with an alternation design. The aim of our research is to improve the already existing solutions and provide an instrument which behaves well in a variety of data scenarios.

The joint use of nonparametric smoothing and permutation theory provides a good solution for the problem of interest; additionally, considering an $M A$ solution, we are able to satisfactorily handle several data scenarios. A simulation study showed that the proposed tool is reliable under the null hypothesis and powerful under the alternative. As regards comparison with an already existing solution, the $M A$ solutions generally behave better than their competitor, always showing an estimated rejection probability under the alternative hypothesis greater than the nominal level of the test; this is not the case, however, for the considered competitor. In general a good feature of the proposed solutions is the increase in their power as the data move further away from the null hypothesis.

The new techniques were also applied to real data, where we confirmed the results of the simulation study and demonstrated the flexibility and usefulness of the methods.

Several further developments can be made using the tools presented here. For instance, it may be of interest to study performances when a smoother other than local regression is used to draw the smoothed time sub-processes, as well as when we simply change the choice of smoothing parameter. The role played by the use of different partial test statistics in the MA solution could also be studied. Furthermore, the performance of the methods could be explored under many other data scenarios, for instance letting the several treatments have also different underlying correlation structures besides the differences in levels or slopes. Another possible kind of presence of carry-over is when there is the effect of the number of treatment administrations that precede a particular administration (see Edgington and Onghena (2007)). Hence two scenarios can happen: the carryover is identical for the several treatments (identical carry-over), or it is different, meaning that the subject's response to a treatment at certain time depends also on the particular treatments that were given at the previous treatment times (differential carry-over). In the former case we do not have any difference among treatments' effects, hence we are under the null hypothesis, and infer- 
ence made through randomization tests in alternation designs is not affected by such a carry-over effect because the effect is constant over all data permutations. In the latter case instead, we are under the alternative hypothesis, as there is a difference in the effects of the several treatments; in this case, inference made through randomization tests is affected by this kind of effect, as this is not constant over all data permutations. It would surely be interesting to check the behavior of the proposed procedure in such case. Another interesting development could go in the direction of interval estimates, trying possible solutions (choices for the estimate of the effect difference) and comparing them in terms of coverage probability.

Acknowledgement. This work has been realized with the support of MIUR project PRIN2008 -CUP number C91J10000000001, within the research Unit at University of Padua and CUP number E61J10000020001, within the research Unit at University of Naples Federico II. Authors wish also to thank the University of Padova (CPDA092350/09) for providing the financial support for this research.

\section{References}

N. Agay, E. Yechiam, Z. Carmel, and Y. Levkovitz. Non-specific effects of methylphenidate (ritalin) on cognitive ability and decision-making of adhd and healthy adults. Psychopharmacology, 210: $511-519,2010$.

A. Baplu. Ritalin, the smart drug: a double-blind placebo controlled n-of-1 experiment in an adolescent with concentration problems. Unpublished masterś thesis, Katholieke Universiteit Leuven, Belgium, 2005.

I. Bulté and P. Onghena. An r package for single-case randomization tests. Behavior Research Methods, 40:467-478, 2008.

I. Bulté and P. Onghena. Randomization tests for multiple-baseline designs: An extension of the scrt-r package. Behavior Research Methods, 41:477-485, 2009.

A. Crutchley and J. A. Temlett. Methylphenidate (ritalin) use and abuse. South African Medical Journal, 89:1076-1079, 1999. 
J.R. de Jong, J.W.S. Vlaeyen, P. Onghena, M.E.J.B. Goossens, M. Geilen, and H. Mulder. Fear of movement/(re)injury in chronic low back pain education or exposure in vivo as mediator to fear reduction. Clinical Journal of Pain, 21:9-17, 2005.

P. Diggle, P. Heagerty, K. Liang, and S. Zeger. Analysis of Longitudinal Data (Second edition). Oxford University Press, USA, New York, 2002.

E.S. Edgington. Randomized single-subject experimental designs. Behavior Research and Therapy, 34:567-574, 1996.

E.S. Edgington and P. Onghena. Randomization Tests, Fourth Edition. Chapman \& Hall, London, 2007.

R. A. Fisher. The design of experiments. Oliver \& Boyd, Edinburgh, 1935.

B.S. Gorman and D.B. Allison. Statistical alternatives for single-case designs. In R.D. Franklin, D.B. Allison, and B.S. Gorman, editors, Design and analysis of single-case research, pages 159214. Lawrence Erlbaum Associates, Mahwah, NY, 1996.

J.R. Levin, L.A. Marascuilo, and L.J. Hubert. $\mathrm{N}=$ nonparametric randomization tests. In T.S. Kratochwill, editor, Single Subject Research: Strategies for Evaluating Change, pages 167-196. Academic, New York, 1978.

P. Onghena. Single-case designs. Encyclopedia of Statistics in Behavioral Science, 4:1850-1854, 2005 .

F. Pesarin. Multivariate permutation tests: with applications in biostatistics. Wiley, Chichester, 2001.

F. Pesarin and L. Salmaso. Permutation Tests for Complex Data: Theory, Applications and Software. Wiley, Chichester, 2010.

J.S. Simonoff. Smoothing Methods in Statistics. Springer-Verlag, New York, 1996.

R. Tannock, R. J. Schachar, R. P. Carr, and G. D. Logan. Dose-response effects of methylphenidate on academic performance and overt behavior in hyperactive children. Pediatrics, 84:648-657, 1989. 
M.M. ter Kuile, I. Bulte', P.T.M. Weijenborg, A. Beekman, R. Melles, and P. Onghena. Therapistaided exposure for women with lifelong vaginismus: A replicated single-case design. Journal of Consulting and Clinical Psychology, 77:149-159, 2009.

B..T. Todman and P. Dugard. Single-Case and Small-n Experimental Designs: A Practical Guide to Randomization Tests. Lawrence Erlbaum Associates, Mahwah, NJ, 2001. 